\title{
Automatic Leptonic Tensor Calculation for Beyond the Standard
}

Model (BSM) Theories

\section{Introduction}

Colossal data output from neutrino experiments (e.g. DUNE, T2HK) will require testing of several BSM theories.

Manual implementation of BSM theories in event generators is time-consuming and prone to errors.

For neutrino events, we can always decompose the squared amplitude $\left(|M|^{2}\right)$ into a hadronic $\left(H^{\mu \nu}\right)$ and a leptonic $\left(L_{\mu v}\right)$ tensor: $|M|^{2}=H^{\mu \nu} L_{\mu \nu}$.

$H^{\mu v}$ is complicated to calculate but event generators are good at doing it. Separation of amplitude into $H^{\mu \nu}$ and $L_{\mu \nu}$ allows easy calculation of effects of BSM theories on $L_{\mu \nu}$. Develop a program to automatically calculate leptonic tensors of BSM theories:

- Requires only BSM Lagrangian.

- Can be easily interfaced to several neutrino event generators.

\section{Methods}

Universal FeynRules Output (UFO) files:

- Use BSM Lagrangian to calculate Feynman vertices. Output in Python.

Lark package

- Parser for string outputs of UFO files.

Berends-Giele algorithm:

- Recursive break down of Feynman diagrams.

- Allows recycling of diagrams' components. Highlyefficient.

- BG Equations: Current $J_{i}(\pi)$ and amplitude $M(\pi)$ for set of particles $\pi$. Base case for $J_{i}(\pi)$ is the particle's wavefunction.

$$
\begin{aligned}
& J_{i}(\pi)=\frac{P_{i}(\pi)}{\downarrow} \sum_{V_{i}^{j, k}, \sum_{P_{2}(\pi)}} \frac{S\left(\pi_{1}, \pi_{2}\right)}{\downarrow} \frac{V_{i}^{j, k}\left(\pi_{1}, \pi_{2}\right)}{J_{\text {Propagator term }}} \frac{J_{j}\left(\pi_{1}\right)}{\downarrow} \frac{J_{k}\left(\pi_{2}\right)}{\downarrow} \\
& \text { Sum over all possible } \\
& \text { vertices and permutations } \\
& M(\pi)=\prod_{\substack{\text { Current for } n \\
\text { Reversed particle } \\
\text { properties }}}^{J_{n}(n)} \cdot \frac{1}{\prod_{\bar{n}}(\pi \backslash n)} \cdot \frac{J_{\bar{n}}(\pi \backslash n)}{l_{\text {Current for }} \bar{n}}
\end{aligned}
$$

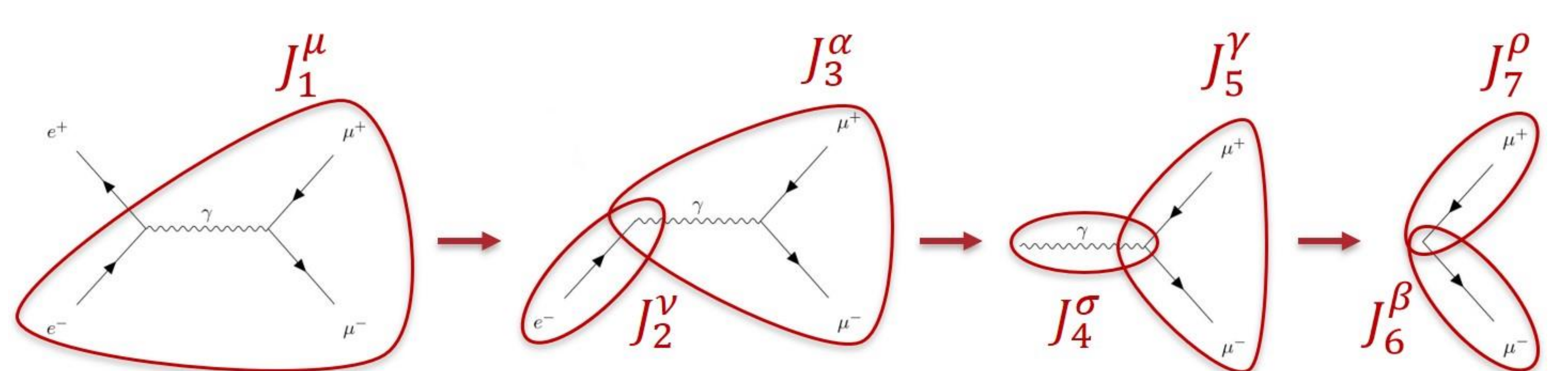

\section{Results and Discussion}

Validation results of squared amplitude of three SM processes $\left(e^{+} e^{-} \rightarrow \mu^{+} \mu^{-}, e^{-} \mu^{-} \rightarrow e^{-} \mu^{-}, e^{+} e^{-} \rightarrow e^{+} e^{-}\right)$plotted versus $\cos (\theta)$ and for randomly generated azimuthal angles $\phi$.

Our results show percentage deviations of order $10^{-14}$ with respect to analytic calculations of our SM processes.

Work can be extended to more complex processes and to BSM theories.

To illustrate how $|M|^{2}$ can be split into $H^{\mu v}$ and $L_{\mu \nu}$, we perform the calculation for $e^{-} N \rightarrow e^{-} N$, with $N$ being an atomic nucleus:

1. Consider simpler case $e^{-} \mu^{-} \rightarrow e^{-} \mu^{-}$with Feynman diagram shown in 2). Diagram is composed by upper $e^{-}$part and lower $\mu^{-}$part.

2. Label $e_{\text {in }}^{-}: 1, \mu_{\text {in }}^{-}: 2, e_{\text {out }}^{-}: 3, \mu_{\text {out }}^{-}: 4$. Matrix element $|M|^{2}$ of $e^{-} \mu^{-}$ scattering given by:

$\underbrace{\frac{2 e^{2}}{\left(p_{1}-p_{3}\right)^{2}} \cdot\left[p_{3 \mu} p_{1 v}+p_{3 \nu} p_{1 \mu}+\left(m_{e}^{2}-p_{1} \cdot p_{3}\right) g_{\mu \nu}-i \epsilon_{\mu \nu \alpha \beta} p_{1}^{\alpha} p_{3}^{\beta}\right]}_{L_{\mu \nu, e^{-}}}$
$\frac{2 e^{2}}{\left(p_{2}-p_{4}\right)^{2}} \cdot\left[p_{4}^{\mu} p_{2}^{v}+p_{4}^{v} p_{2}^{\mu}+\left(m_{\mu}^{2}-p_{2} \cdot p_{4}\right) g^{\mu \nu}+i \epsilon^{\mu \nu \alpha \beta} p_{2 \alpha} p_{4 \beta}\right]$
$L_{\mu^{-}}^{\mu \nu}$

3. Similarly, matrix element $|M|^{2}$ of $e^{-} N$ scattering contains the upper $e^{-}$part and, thus, $L_{\mu v, e^{-}}$.Then:

$$
|M|^{2}=L_{\mu \nu, e^{-}} H_{N}^{\mu \nu}
$$

Where $H_{N}^{\mu v}$ is the hadronic tensor. Depending on the energy, $N$ might be the nucleus itself, a nucleon or a parton inside a nucleon.

\section{Conclusion and Future Steps}

Validation results are promising, proving our method works for SM processes.

Convert amplitude into leptonic tensor to be interfaced with event generators.

Perform tests in DIS events as well as with some BSM theories using leptonic tensor.

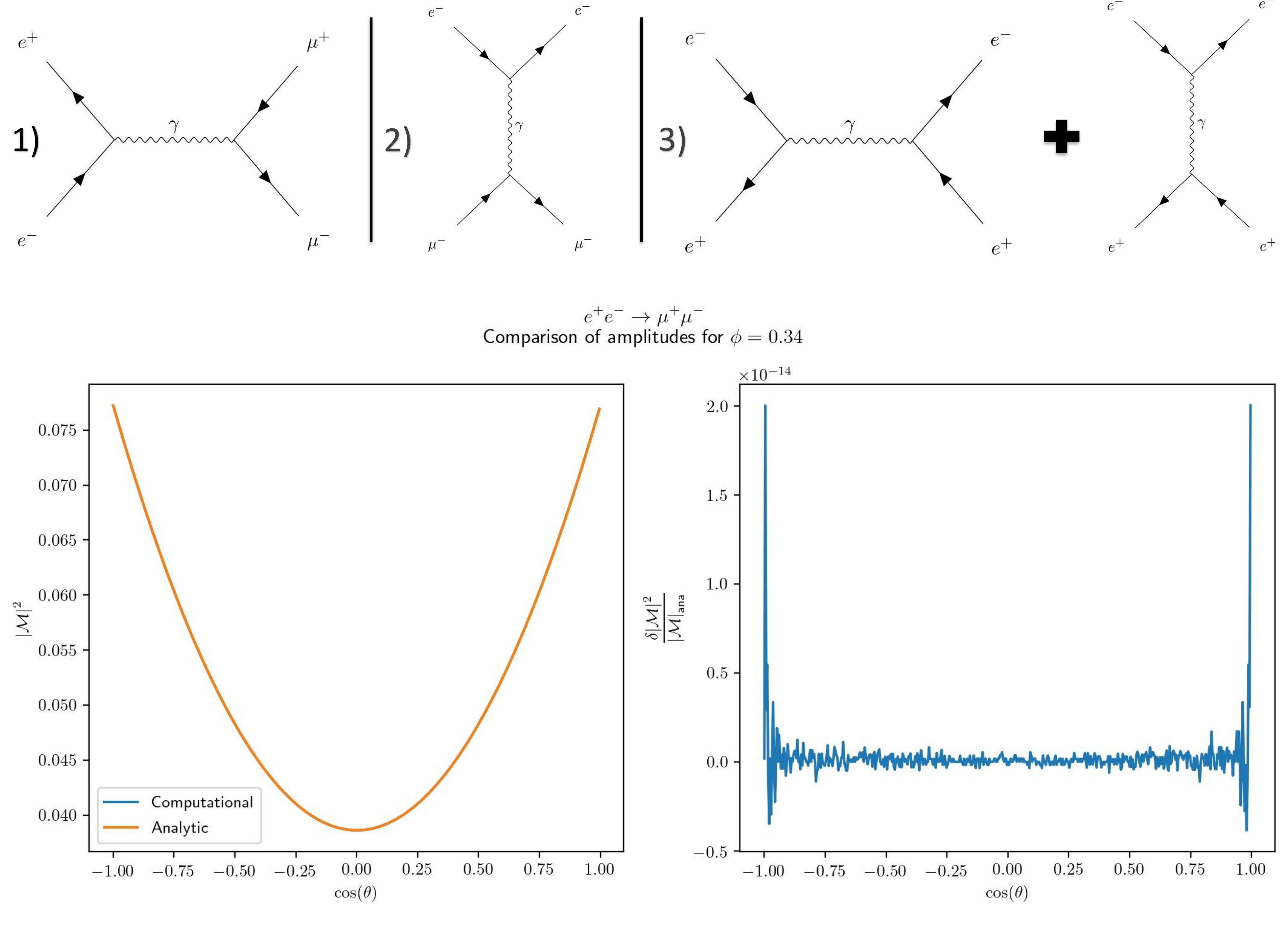

Comparison of our computational and analytic squared amplitudes for $e^{+} e^{-} \rightarrow \mu^{+} \mu^{-}$

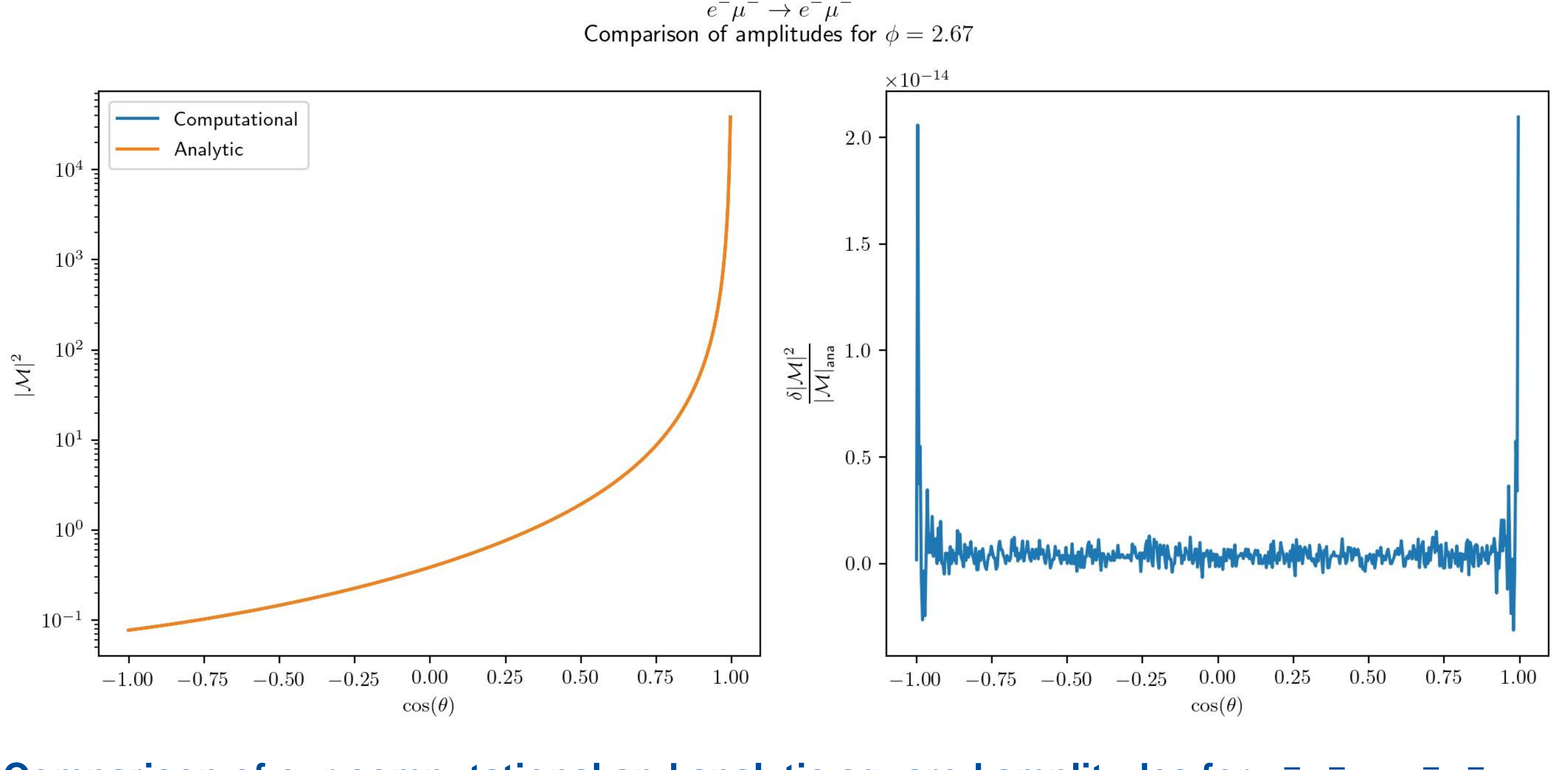

Comparison of our computational and analytic squared amplitudes for $e^{-} \mu^{-} \rightarrow e^{-}$ as a function of $\cos (\theta)$ for a (random) value of $\phi=2.67 \mathrm{rad}$.

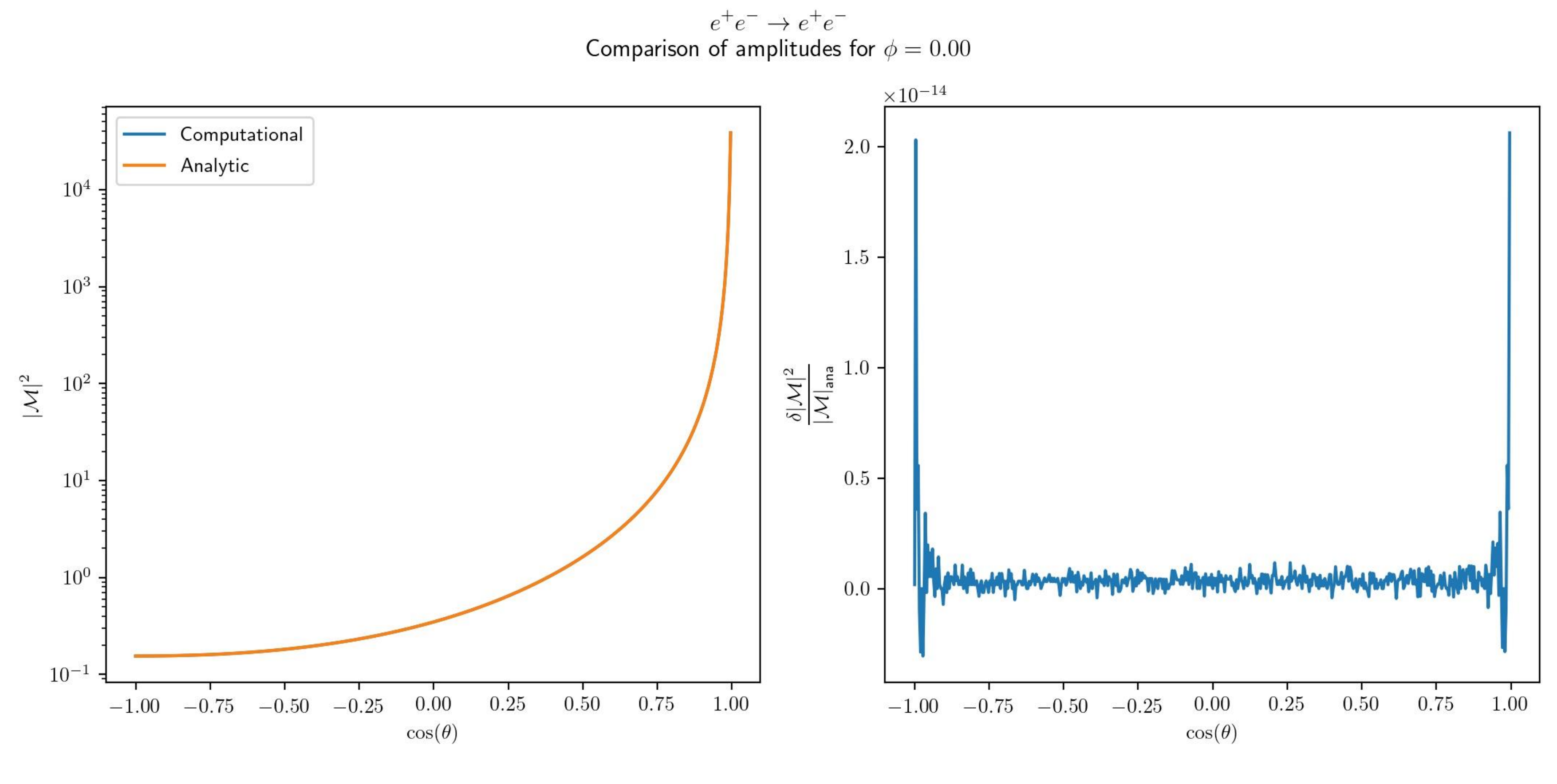
Comparison of our computational and analytic squared amplitudes for $e^{+} e^{-} \rightarrow e^{+} e^{-}$as 